\title{
Identification of nuclear localization signal and nuclear export signal of VP1 from the chicken anemia virus and effects on VP2 shuttling in cells
}

Jai-Hong Cheng ${ }^{1 *}$ (D) Guan-Hua Lai ${ }^{2}$, Yi-Yang Lien ${ }^{3,4}$, Fang-Chun Sun ${ }^{5}$, Shan-Ling Hsu' ${ }^{6}$ Pei-Chin Chuang ${ }^{1}$ and Meng-Shiou Lee 7* $^{*}$

\begin{abstract}
Background: VP1 of the chicken anemia virus (CAV) is a structural protein that is required for virus encapsulation. VP1 proteins are present both in the nucleus and cytoplasm; however, the functional nuclear localization signal (NLS) and nuclear export signal (NES) of VP1 are still unknown. This study aimed to characterize the NLS and NES motifs of VP1 using bioinformatics methods and multiple-site fragment deletions, and investigate shuttling of VP2 from nucleus to cytoplasm by co-transfection with VP1.

Methods: Two putative NLS motifs were predicted by the WoLF PSORT and NLStradamus programs from the amino acid sequence of VP1. Three NES motifs of VP1 were predicted by the NetNES 1.1 Server and ELM server programs. All mutants were created by multiple-site fragment deletion mutagenesis. VP1 and VP2 were coexpressed in cells using plasmid transfection.

Results: A functional NLS motif was identified at amino acid residues 3 to 10 (RRARRPRG) of VP1. Critical amino acids 3 to 10 were significantly involved in nuclear import in cells and were evaluated using systematic deletion mutagenesis. Three NES motifs of VP1 were predicted by the NetNES 1.1 Server and ELM server programs. A functional NES was identified at amino acid residues 375 to 388 (ELDTNFFTLYVAQ). Leptomycin B (LMB) treatment demonstrated that VP1 export from nucleus to cytoplasm occurred through a chromosome region maintenance 1 (CRM1)-dependent pathway. With co-expression of VP1 and VP2 in cells, we observed that VP1 may transport VP2 from nucleus to cytoplasm.

Conclusion: Our data showed that VP1 of CAV contained functional NLS and NES motifs that modulated nuclear import and export through a CRM1-dependent pathway. Further, VP1 may play a role in the transport of VP2 from nucleus to cytoplasm.
\end{abstract}

Keywords: Chicken anemia virus, VP1, VP2, Nuclear localization signal, Nuclear export signal, CRM1-dependent pathway

\footnotetext{
*Correspondence: cjh1106@cgmh.org.tw; leemengshiou@mail.cmu.edu.tw ${ }^{1}$ Center for Shockwave Medicine and Tissue Engineering, Department of Medical Research, Kaohsiung Chang Gung Memorial Hospital and Chang Gung University College of Medicine, 123 Tai-Pei Road, Niao Sung District, Kaohsiung, Taiwan833

${ }^{7}$ Department of Chinese Pharmaceutical Science and Chinese Medicine Resources, China Medical University, 91, Hsueh-Shih Road, Taichung, Taiwan Full list of author information is available at the end of the article
}

(c) The Author(s). 2019 Open Access This article is distributed under the terms of the Creative Commons Attribution 4.0 International License (http://creativecommons.org/licenses/by/4.0/), which permits unrestricted use, distribution, and reproduction in any medium, provided you give appropriate credit to the original author(s) and the source, provide a link to the Creative Commons license, and indicate if changes were made. The Creative Commons Public Domain Dedication waiver (http://creativecommons.org/publicdomain/zero/1.0/) applies to the data made available in this article, unless otherwise stated. 


\section{Background}

The chicken anemia virus (CAV) is a small virus of 23 to $25 \mathrm{~nm}$ in size; it is an icosahedral, non-enveloped virus, resistant to heat, lipid solvents and disinfectants [1]. CAV belongs to the genus Gyrovirus of the Anelloviridae family. The viral genome is a single-stranded DNA that is circular and covalently linked, consisting of approximately 2300 base pairs in its replicative form [2]. In 1979, Dr. Yuasa reported that vaccines contaminated with CAV had been distributed worldwide, causing spread of the disease [3]. CAV infection results in large economic damage, as the clinical disease contributes to vertical transmission and immune dysfunction in combination with other pathogens [4].

The CAV genome replicates through the rolling circle method [5]. The genomic DNA encodes three viral proteins (VP1, VP2 and VP3) from a 2.1-kb transcript [6]. Among the viral proteins, VP1 is a capsid protein (51 $\mathrm{kDa})$ and VP2 is a non-structural protein $(24 \mathrm{kDa})$, containing a dual-specific phosphatase activity and acting as a scaffold protein in the capsid assembly. VP1 and VP2 are protective proteins that induce neutralizing antibodies [7]. VP3 is called "apoptin"; it is the smallest of the three viral proteins at $13 \mathrm{kDa}$, with a unique apoptotic-inducing property $[6,8]$.

In the life cycle of the virus, the subcellular localization of viral proteins, especially in the nucleus, may contribute to cell apoptosis, viral replication or host cell proliferation $[9,10]$. Thus, determination of the nuclear localization signal (NLS) and nuclear exporting signal (NES) of a viral protein is critical, and is required in order to reveal the importance of viral proteins in virus replication in cells. Recently, three CAV viral proteins, VP1, VP2 and VP3, have been found to possess nuclear localization activity in cells [11-14]. When GFP-VP1 and GFP-VP2 are transiently expressed in cells, VP1 and VP2 are observed to be present throughout the nucleoplasm [11]. VP3 has been reported to trigger cell apoptosis, and has been found to aggregate within the nucleus by phosphorylation of Threonine 108 [13, 14]. These results suggested that a functional NLS may be present in all three CAV viral proteins. To date, the functional NLSs of VP2 and VP3 have been demonstrated via bioinformatics and biochemical experiments [12-15]. VP3 contains a bipartite-type NLS and NES, suggesting potential shuttling of the protein between nucleus and cytoplasm $[14,15]$. VP2 is a CRM1-independent nuclear protein with a simple NLS spanning amino acid residues from 133 to 138, and may not have a NES motif [12]. Moreover, VP2 and VP3 expressions have indicated protein-protein interaction in the cell [16]. Further, the apoptosis ability of VP3 is down-regulated directly by VP2 phosphatase through de-phosphorylation of Threonine 108 of VP3 [14]. VP1 is the only structural protein of CAV, and is required theoretically for packaging and formation of infectious particles [17]. A polyclonal chicken anti-CAV sera recognized all strains that have been tested, but only monoclonal antibody 2A9 has been mapped with VP1 [18-20]. The formation of epitopes recognized by polyclonal virus-neutralizing $(\mathrm{VN})$ chicken antibodies requires co-expression of VP1 and VP2, implying that VP2 acts as a scaffold protein with VP1 [7, 21]. This finding was subsequently proved by analysis of VP1VP2 protein-protein interaction [16]. In spite of this finding, the possible functional domain of VP1, especially in terms of the cellular localization, is not well understood, although some researchers have demonstrated the subcellular distribution of VP1 in cells using transient protein expression and immunofluorescence assays $[11,22]$. The $\mathrm{N}$-terminus of VP1 has been reported to possess a cell-penetrating activity [23]. Therefore, further research into and analysis of protein trafficking of VP1 may provide more information for elucidation of the biological function of nuclear localization of CAV viral proteins in virus replication.

In the present study, we used bioinformatics methods to predict putative NLS and NES motifs and identified the functional NLS and NES by performing experiments. Differing versions of VP1 were created by truncation mutagenesis, and the locations of functional NLS and NES sequences in VP1 were confirmed. Further, leptomycin B (LMB), an inhibitor of CRM1, was used to identify the function of the NES motif in the VP1 protein [15]. Finally, when VP1 and VP2 were co-expressed in cells, VP1 was observed to modulate the subcellular redistribution of VP2 from nucleus to cytoplasm. To the best of our knowledge, this is the first report to describe the functional NLS/NES motifs of VP1 and the interaction between VP1 and VP2 proteins allowing VP2 export from nucleus to cytoplasm.

\section{Methods \\ Construction of multiple-site fragment deletions}

For the expression of truncated VP1-GFP fusion proteins, the full-length VP1 plasmid (pGEX-6P-1-VP1) was used as a template to amplify fragments by polymerase chain reaction (PCR) with primers (Table 1) [24]. After agarose gel purification, PCR products were ligated into the $y T \& A$ vector (Yeastern Biotech, Taiwan). The PCR fragments were released from the $\mathrm{YT} \& \mathrm{~A}$ vector by EcoRI/XhoI digestion and then inserted into pcDNA3.1-GFP [12].

\section{Analysis of amino acid sequence of VP1}

Five isolates of CAV VP1, including Australia/CAU269-7/ 2000 (accession number: Q9IZU5), Germany Cuxhaven-1 (accession number: Q99153), Japan 82-2 (accession number: P54090), USA 26p4 (accession number: P54089), and USA CIA-1 (accession number: P54088), were identified 
Table 1 The primers were used to construct the various truncated, internal deletion mutants by PCR

\begin{tabular}{|c|c|c|c|}
\hline Primer name & Type & Length & Sequence $\left(5^{\prime}-3^{\prime}\right)$ \\
\hline VP1 EcoR1 & Forward & 25 -mer & AGAATTCATGGCAAGACGAGCTCGC \\
\hline VP1 Xho1 R & Reverse & 20-mer & TCCTCGAGGGGCTGCGTCTC \\
\hline VP1 ND9 EcoR1 & Forward & 24-mer & AGAATTCATGGGCCGATITTACGC \\
\hline VP1 DN19 EcoR1 & Forward & 26-mer & AGAATTCATGCACAACCTCAAGCGAC \\
\hline VP1 ND30 EcoR1 & Forward & 25-mer & AGAATTCAAATTTCGCCATCGCCGC \\
\hline VP1 ND60 EcoR1 & Forward & 25-mer & AGAATTCCTGCCGAACCCGCAGAGC \\
\hline VP1 ND129 EcoR1 & Forward & 25-mer & AGAATTCGGCGAACTGATTGCGGAT \\
\hline VP1 CD10 Xho1 & Reverse & 21-mer & TCCTCGAGGCCTCTCGGTCTG \\
\hline VP1 CD103 Xho1 & Reverse & 24-mer & TCCTCGAGGGCGACTCTCGGCCCC TAAGATGG \\
\hline VP1 CD126 Xho1 & Reverse & 22-mer & TCTCGAGTCCGATITTGCTCAC \\
\hline VP1 internal NES deletion 1162 & Forward & 24-mer & GCGGCCGCGGCACAAATAAGTCGC \\
\hline VP1 internal NES deletion 1119 & Reverse & 27-mer & GCGGCCGCATGATGCGGGTGCACT \\
\hline
\end{tabular}

in the Universal Protein Resource (UniProt). All sequences were aligned and analyzed using CLUSTAL W and MView in Biology Workbench 3.2 (San Diego Supercomputer Center; SDSC) $[25,26]$ and compared with Taiwan CIA-89. The putative NLS motifs of VP1 were predicted by WoLF PSORT [27] and NLStradamus [28], and the putative NES motifs were predicted by the NetNES 1.1 Server [29] and ELM server [30].

\section{Plasmid transfection}

Chinese hamster ovary ( $\mathrm{CHO})-\mathrm{K} 1$ cells were used for plasmid transfection. GIBCO ${ }^{\circ}$ Dulbecco's Modified Eagle Medium: Nutrient Mixture F-12 (DMEM/F-12) (Invitrogen, USA) was supplemented with $10 \%(v / v)$ fetal bovine serum (FBS) (GIBCO/Invitrogen, USA), 100 units $/ \mathrm{mL}$ penicillin, and $100 \mu \mathrm{g} / \mathrm{mL}$ streptomycin for cell culture. A 24-well culture plate was seeded with $9 \times 10^{4}$ cells per well for transfection. The plasmids were transfected into $\mathrm{CHO}$ cells using FuGENE ${ }^{\bullet} 6$ transfection reagent (Promega, USA) according to the manufacturer's instructions in order to observe the localization of VP1-GFP and various deletion mutants. For transfection of MDCC-MSB1 cells, $4 \times 10^{6}$ cells were gently pipetted with $15 \mu \mathrm{g}$ of pEGFPVP1, pc-mCherry-vp2 and pEGFP-VP3 in serum-free RPMI 1640 medium separately. Each mixture was then transferred into a 0.4-cm gap electroporation cuvette, and the cuvette was harvested on ice for $5 \mathrm{~min}$. Electroporation of MDCC-MSB1 cells was performed using a Gene Pulser II (Bio-Rad, USA) with a Time Constant Protocol set at 34 milliseconds and an operating voltage of $300 \mathrm{~V}$. After electroporation, the transfected cells were cultured in complete medium in a 6-well plate for 24 to $48 \mathrm{~h}$. Post-transfection, the expressions of recombinant EGFPVP1, pc-mCherry-vp2 and pEGFP-VP3 proteins were analyzed by confocal fluorescence microscopy to ensure that transfection was effective.

\section{Leptomycin B treatment}

$\mathrm{CHO}$ cells were seeded at a density of $9 \times 10^{4}$ cells per well in 24-well culture plates for CRM1-dependent protein assay. In the experiments, cells were treated with fresh medium containing $20 \mathrm{ng} / \mathrm{mL} \mathrm{LMB} \mathrm{(+)} \mathrm{(Calbio-}$ chem, Germany) or with phosphate-buffered saline (PBS) as the LMB (-) control. After one hour of incubation, cells were fixed, washed and stained with DAPI [12]. Images of GFP fluorescence and DAPI were viewed and captured using a ZEISS AXIOVERT 200 microscope and an AxioCam HRm CCD camera, respectively.

\section{Sample preparation for confocal microscopy}

Plasmids of pEGFP-VP1, pc-mCherry-vp2 and pEGFP-VP3 were constructed as described in a previous study [14]. MDCC-MSB1 cells were transfected with plasmids pEGFP-VP1, pc-mCherry-vp2 and pEGFP-VP3. Fluorescent images were captured using a confocal fluorescence microscope to observe protein fluorescence to verify the preparation of EGFP-expressing cells and EGFP-VP1, EGFP-VP3 and mCherry-vp2-expressing cells. Transfected cells were collected and fixed with $4 \%$ formaldehyde in the dark. After washing the fixed cells twice to remove residual formaldehyde, cells were stained in $0.1 \%$ PBS-T with $1 \mu \mathrm{g} / \mathrm{mL}$ DAPI for $5 \mathrm{~min}$ at $37^{\circ} \mathrm{C}$ in the dark. Then, the stained cells were mounted with gelvatol medium (Sigma, USA) on a glass slide for confocal microscope observation. Confocal laser scanning microscope (CLSM) images were captured using a Leica TCS SP8 confocal microscope, and the images were integrated using LAS X Leica Confocal Software.

\section{Results}

Revisiting the distribution of VP1 in mammalian cells

In previous studies, VP1 has been shown to be distributed in the nucleus and cytoplasm, indicating that putative NLS and NES motifs of VP1 were functional in plant or chicken cells. To further revisit the subcellular 
(USA CIA-1) and 97.8\% (Japan 82-2) as compared with Taiwan CIA-89.

Computational prediction of protein localization from the amino acid sequence was an important step in the present study. Numerous computational methods are available for analysis of the protein sequence to predict the most likely locations, such as in the nucleus or in the cytoplasm. Therefore, we used the WoLF PSORT and NLStradamus programs to predict the NLS sequence from the full-length amino acid sequence of VP1 (Taiwan CIA-89) (Figs. 1b and 2). Two NLS motifs (designated NLS1 and NLS2) were predicted by the WoLF PSORT program. The putative NLS motifs spanned amino acid residues from 3 to 19 (NLS1) and 24 to 47 (NLS2). On the other hand, putative NES motifs were identified by the NetNES 1.1 Server and ELM server (Fig. 2). Three putative NES motifs spanning amino acid residues 76 to 84 (NES1), 109 to 119 (NES2) and 375 to 387 (NES3) were pinpointed. In light of these results, further investigations were needed in order to elucidate whether the putative NLS and NES were functional in VP1.

\section{Functional NLS of VP1}

Using bioinformatics analysis, two putative NLS motifs (NLS1 and NLS2) were predicted to be present in the N-terminal of VP1 (Fig. 2). Hence, in order to determine the functional site of the NLS motif in VP1, a full-length clone and six deletion clones of VP1 were created to fuse with GFP at the C-terminal (Fig. 3a). The subcellular locations of these expressed constructs were examined in the transfected cells based on the GFP distribution patterns at $48 \mathrm{~h}$ posttransfection. The serious truncated N-terminal deletions of VP1-GFP identified for NLS analysis were VP1-ND129, VP1-ND60, VP1-ND30, VP1-ND19 and VP1-ND9. The green fluorescence of these truncations was predominantly distributed in the cytoplasm of $\mathrm{CHO}$ cells (Fig. 3b). These results suggested that the NLS motif of VP1 was monopartite, presented between the 3rd and the 9th amino acid in the sequence. Furthermore, VP1-CD10 was tested, and the GFP was found to be localized in the nucleus of $\mathrm{CHO}$ cells (Fig. 3b).

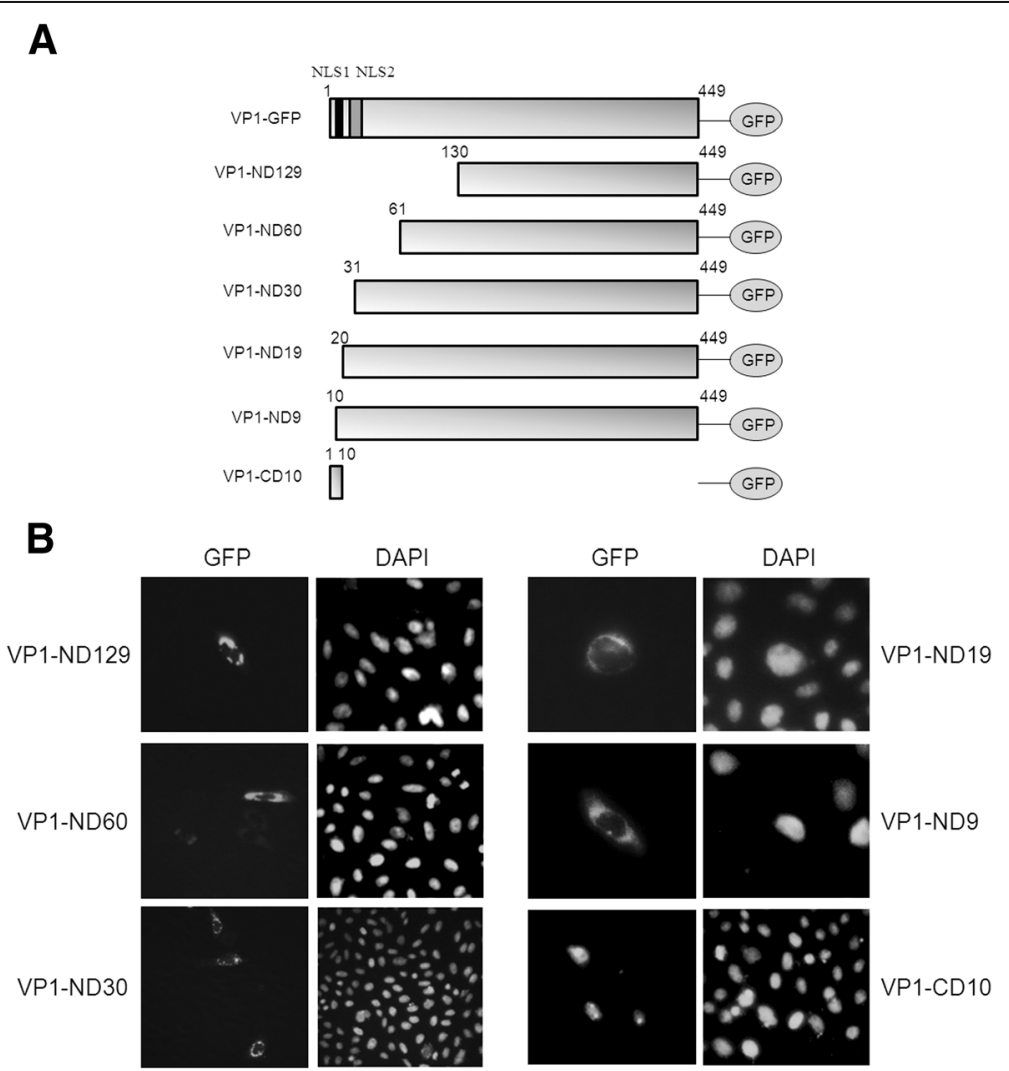

Fig. 3 NLS motif characterized by multiple-site fragment deletions. a Truncated fragments of VP1 (gray bars) encoding constructs fused with GFP (oval shape) were used in this study, including VP1-ND129, VP1-ND60, VP1-ND30, VP1-ND19, VP1-ND9 and VP1-CD10. The putative NLS1 (black box) and NLS2 (gray box) are shown in the full length of VP1 (gray bar). The numbers of amino acid residues are presented in the constructs. $\mathbf{b}$ Subcellular localization of N-terminal deleted VP1-GFP constructions (VP1-ND129, VP1-ND60, VP1-ND30, VP1-ND19 and VP1-ND9) and C-terminal deleted VP1-CD10 are shown in CHO cells. All cells were fixed and stained with DAPI. The distributions of the truncated VP1-GFP constructs in the cells were observed using fluorescence microscopy (400x magnification) 


\section{NES motif in VP1}

NES in capsid protein VP1 of the CAV positive-strand DNA virus is not well-characterized; nor is the pathway that determines its export from nucleus to cytoplasm. Therefore, we characterized the computational prediction of NES motifs within VP1. Three putative NES motifs were identified, spanning amino acid residues 76 to 84 (NES1), 109 to 119 (NES2) and 375 to 387 (NES3) in VP1 (Fig. 1b and Fig. 2).

In order to verify functionally-significant NES motifs, we created various truncated mutants of VP1GFP (Fig. 4a). After transfection, we observed that the C-terminal-truncated mutants (VP1-CD103 and VP1-CD126) were distributed in the nucleus (Fig. 4b). This result indicated that NES1 and NES2 were not NES motifs and could not assist in truncated VP1 export to the nucleus. The results also showed that the $\mathrm{N}$-terminal region of VP1 (amino acid 1 to 126) did not contain an NES motif. Further, we created an internal deletion mutant, VP1-IDNES3, to verify the function of NES3 (Fig. 4a and b). VP1-IDNES3 was located in the nucleus after transfection. We compared the distribution of VP1-GFP and VP1-IDNES3 in the cells, and the results suggested that NES3 was the NES motif (Fig. 4b).

\section{VP1 is a CRM1-dependent protein}

Typically, the nuclear protein containing NES motifs can shuttle between nucleus and cytoplasm. In order to evaluate the functional NES3 motif, we used LMB to interfere with CRM1-NES interaction and verified the functionality of the NES motif (Fig. 4c). Functional NES3 was investigated using VP1-GFP, as shown in Fig. 4c, together with LMB $(+)(20 \mathrm{ng} / \mathrm{mL})$. The distribution of green fluorescence was observed, and the results demonstrated that LMB was able to affect the nuclear export of VP1. Therefore, it was evident that VP1 nuclear export was a CRM1-dependent process and that there was a functional NES motif present in VP1.

\section{Nucleo-cytoplasmic shuffling of VP2 is triggered by VP1}

In a previous study, VP2 exhibited nuclear localization activity and did not contain an NES motif. In contrast, when VP1 was transiently-expressed in MDCC-MSB1 cells, the subcellular distribution of VP1 was observed to be in the nucleo-cytoplasmic compartment, and this result was consistent with the subcellular localization of VP1 in CHO cells (Figs. 1 and 5a). Notably, however, VP2 was exported from the nucleus into the cytoplasmic compartment when transient co-expression of VP2 and VP1 was attained, but not with co-expression of VP2

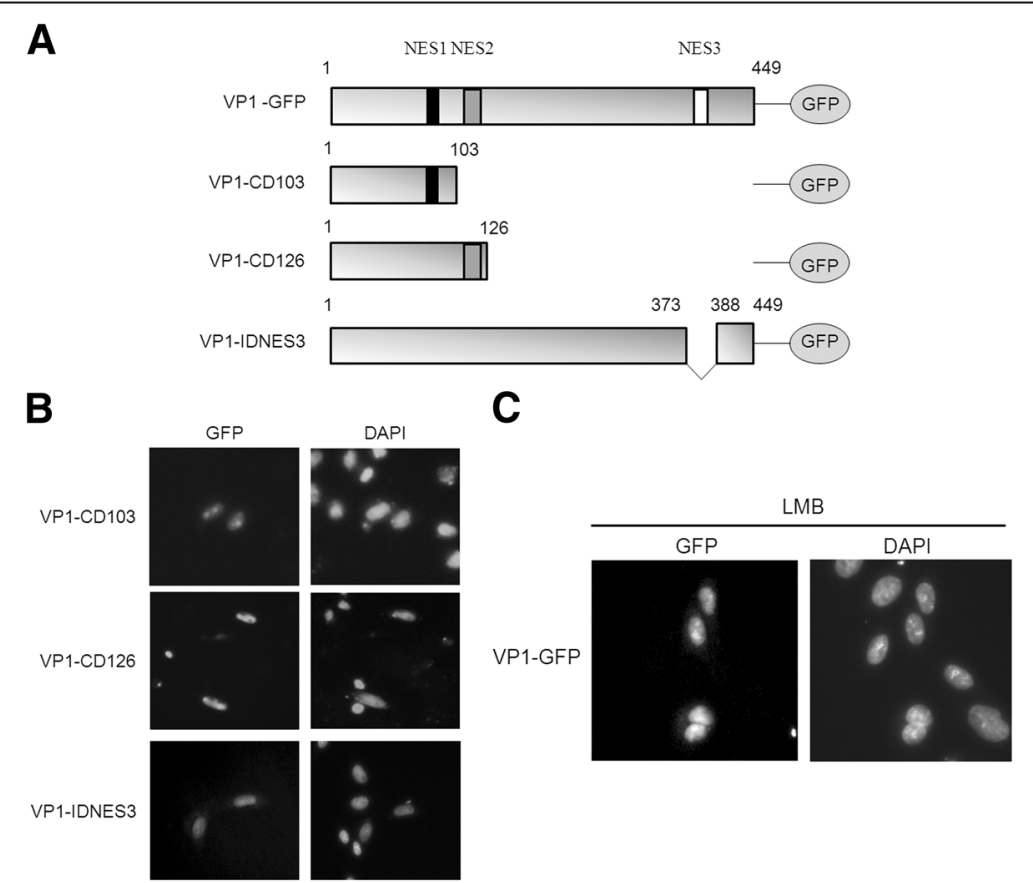

Fig. 4 The NES motif of VP1 was characterized and found to be CRM1-dependent for nuclear export to the cytoplasm. a Truncated constructions of VP1-GFP were used to identify the NES motif. Truncated fragments of VP1 (gray bars) encoding constructs fused with GFP (oval shape) were used in this study, including VP1-CD103, VP1-CD126 and VP1-IDNES3. The putative NES1 (black box), NES2 (gray box) and NES3 (white box) are shown in VP1 (gray bars). The numbers of amino acid residues are presented in the constructs. b Subcellular localization of C-terminal deleted VP1-GFP constructions (VP1-CD103 and VP1-CD126) and internal deleted VP1-IDNES3 are shown in CHO cells. All cells were fixed and stained with DAPI. b VP-GFP was treated with LMB $(20 \mathrm{ng} / \mathrm{mL})$ after $48 \mathrm{~h}$ of transfection. The distributions of the wild-type VP1-GFP and the truncated VP1-GFP in the cells were observed using fluorescence microscopy (400x magnification) 


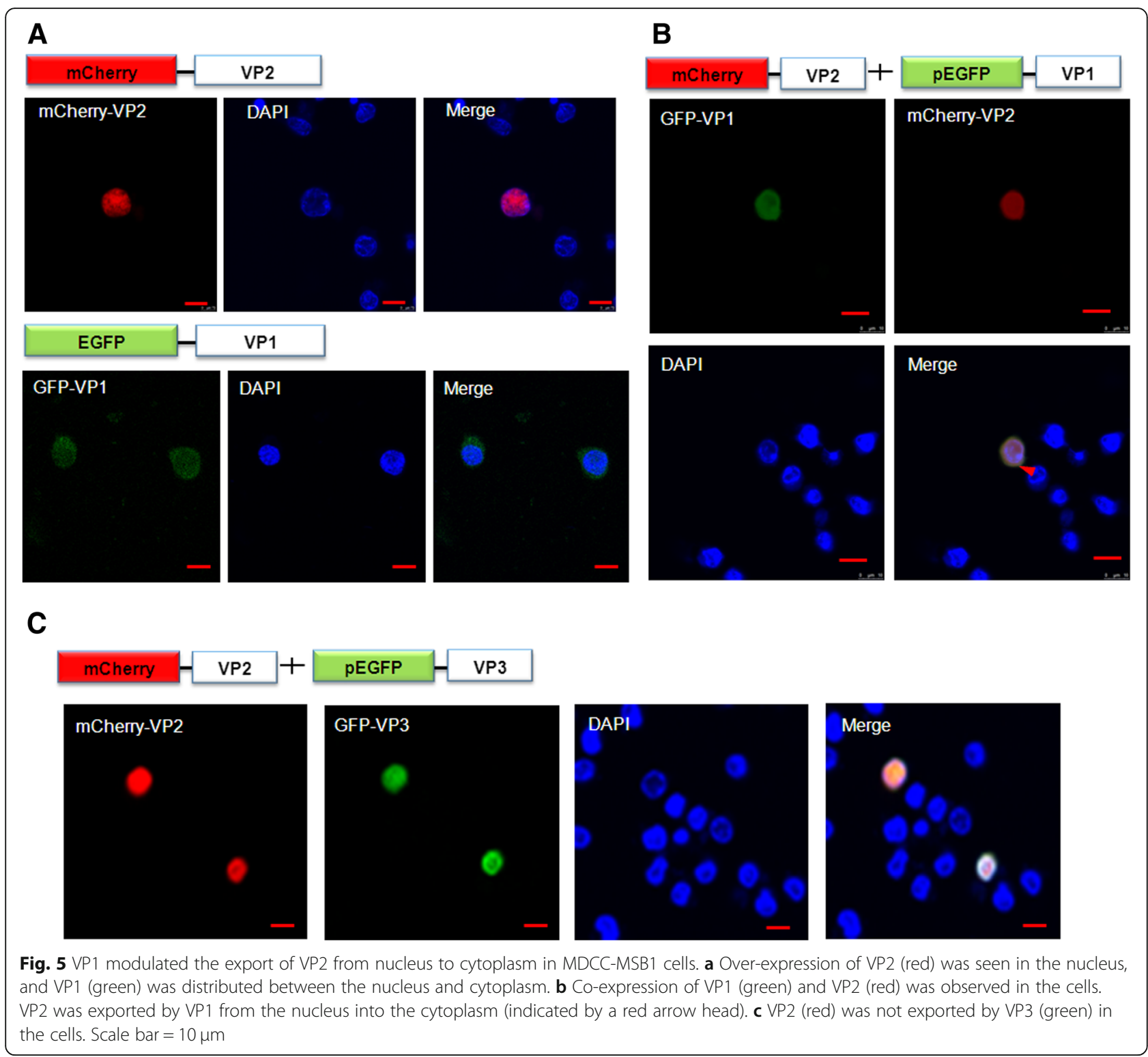

and VP3 (Fig. 5b and c). Further, the subcellular distribution of VP1 did not change in MDCC-MSB1 cells when VP2 was co-expressed at the same time. Taking these results together, VP1 displayed an interaction with VP2 in terms of co-localization of VP1 and VP2 in cells, and VP2 export from nucleus to cytoplasm was triggered by VP1.

\section{Discussion}

Young chickens infected with CAV suffer anemia, hemorrhaging, and immunosuppressive diseases, and the mortality of the chickens is increased [1, 3, 31]. The virus contains a very compressed genomic organization, and three viral proteins (VP1, VP2 and VP3) translated from a single polycistronic 2.1-kb mRNA have been identified in virus-infected cells $[3,6]$. VP1 is the only structural protein that has a very basic $\mathrm{N}$-terminal region that encapsulates a negative-strand DNA genome [32]. The C-terminal region of the protein carries a conserved motif for rolling circle replication (RCR) [33, 34]. In this study, CAV VP1 was observed in the nucleus and cytoplasm in transfected $\mathrm{CHO}$ and MDCC-MSB1 cells (Figs. 1 and 5). Sequence analysis indicated that the $\mathrm{N}$-terminus of the CAV VP1 protein contains abundant basic amino acid residues (Fig. 2). Investigation of the subcellular localization of the truncated CAV VP1 proteins fused with GFP indicated that the 7 amino acids at the $\mathrm{N}$-terminus were crucial and sufficient to direct the accumulation of the protein in the nuclei (Fig. 3), and amino acid residues from 375 to 387 exhibited a functional NES motif (Fig. 4). Dr. Hu reported that the $\mathrm{N}$-terminus of the VP1 protein consists of enriched arginine residues similar to HIV-TAT [23]. The sequence 
has an efficient cell-penetrating activity. However, that study did not identify the sequence systemically to show that it is the NLS of VP1. The other viral protein, VP3, is a nucleocytoplasmic shuttling protein, the localization of which is mediated by an N-terminal NES and a C-terminal NLS $[13,15]$. Although CAV VP1 and VP3 were shuttled between nucleus and cytoplasm during virus infection, VP2 was restricted to the nuclei in plasmid-transfected cells [12].

In addition, VP1 interacts with VP2, which is a nonstructural protein exhibiting phosphatase activity, and the dual specificities are indispensable for CAV replication [21, 35-37]. VP2 has been found to contain a functional NLS and is a CRM1-independent protein [12]. Further, VP2 also associates with mini-chromosome maintenance protein 3, a component of DNA pre-replication complexes [12]. In this study, under co-transfection of VP1 and VP2, it was observed that VP2 might be shuffled by VP1 between nucleus and cytoplasm in cells. Dr. Sun reported that a specific interaction domain between VP1 and VP2 was observed by yeast two-hybrid system [16]. As VP2 lacks an NES motif, VP1 may act as a nuclear exporting factor with VP2 for nucleo-cytoplasmic shuffling in cells. There may be other hosts involved in the export of VP2 by VP1 from nucleus to cytoplasm for regulation of the viral life cycle or hosting cell proliferation. Molecules that interact with VP2 in the cytosol require further investigation in the future.

NES is a short, leucine-rich motif that is an export substrate of CRM1 and identifies in HIV Rev. and protein kinase A inhibitor [38, 39]. The function of NES is important for the virus during the viral life cycle and plays a vital role in the replication, assembly, and budding of viruses. For example, the influenza A virus matrix 1 protein (M1) is specifically dependent on the Flu-A-M1 NES and is critical for influenza A virus replication [40]. CRM1-mediated nuclear export has been reported to be involved in several viral infections, including the influenza A virus [40, 41], human papillomavirus [42] and HIV [38, 43]. Our data indicated that CAV VP1 utilized a CRM1-dependent nuclear export pathway, as well as VP3 (Fig. 4b). For the previously-identified NES, only two leucines located in VP3 contributed to the activity of this motif [44]. Only two leucines (Leu376 and Leu383) have been discovered in the VP1 NES, and a number of leucine-rich NESs have been characterized, in which one or two leucine residues contribute to the nuclear export activity. Taken together, the nucleocytoplasmic shuttling activity suggested that VP1 may coordinate with other viral proteins such as VP2 and VP3 to complete the viral life cycle.

\section{Conclusion}

In the current study, VP1 of CAV was found to contain a functional NLS motif that spanned amino acids 3 to 9 of the protein. Further examination showed that VP1 also has an NES motif spanning amino acids 375 to 387 at the C-terminus. VP1 exhibited sensitivity to the CRM1 inhibitor LMB, and was found to be a CRM1-dependent protein. Characterization of the NLS and NES of the CAV VP1 protein provided the basis for further research. This study also explored the role of nuclear involvement of the VP1 protein in the development of CAV infection and the biological implication of its shuttling between nucleus and cytoplasm.

\begin{abstract}
Abbreviations
CAV: Chicken anemia virus; $\mathrm{CHO}$ : Chinese hamster ovary; CRM1: Chromosome region maintenance 1; DMEM/F-12: Dulbecco's Modified Eagle Medium: Nutrient Mixture F-12; FBS: Fetal bovine serum; LMB: Leptomycin B; NES: Nuclear export signal; NLS: Nuclear localization signal; PBS: Phosphate-buffered saline; RCR: Rolling circle replication
\end{abstract}

\section{Acknowledgements}

The authors would like to thank Prof. Min-Ying Wang (Graduate Institute of Biotechnology, National Chung Hsing University, Taichung, Taiwan) for kindly providing the pcDNA3.1-GFP plasmid. We also thank the Medical Research Core Facilities Center, Office of Research \& Development at China Medical University (Taichung, Taiwan, R.O.C.) for supporting experiments and data analysis on confocal microscopic observation and flow cytometry.

\section{Funding}

This research was supported by grants from the Chang Gung Medical Foundation and the Ministry of Science and Technology of Taiwan (Grant nos. CMRPG8F1131; NSC-95-2313-B-039-004-; NSC-96-2313-B-276-001-MY3).

Availability of data and materials

Not applicable.

Authors' contributions

JHC and MSL conceived and designed this study. JHC and GHL contributed to the majority of the plasmid construction and cell experiments. JHC, YYL, FCS, and MSL helped with the laboratory experiments and interpreted the data. JHC and MSL prepared the manuscript. JHC, YYL, FCS, PCC and MSL reviewed and critically revised the manuscript. All authors read and approved the final manuscript.

Ethics approval and consent to participate Not applicable.

Consent for publication

Not applicable.

\section{Competing interests}

The authors have no conflicts of interest to declare.

\section{Publisher's Note}

Springer Nature remains neutral with regard to jurisdictional claims in published maps and institutional affiliations.

\footnotetext{
Author details

${ }^{1}$ Center for Shockwave Medicine and Tissue Engineering, Department of Medical Research, Kaohsiung Chang Gung Memorial Hospital and Chang Gung University College of Medicine, 123 Tai-Pei Road, Niao Sung District, Kaohsiung, Taiwan833. ${ }^{2}$ Graduate Institute of Biotechnology, College of Agriculture and Natural Resources, National Chung Hsing University, Taichung 40402, Taiwan. ${ }^{3}$ Department of Veterinary Medicine, National Pingtung University of Science and Technology, Pingtung, Taiwan. ${ }^{4}$ Research Center of Animal Biologics, National Pingtung University of Science and Technology, Pingtung, Taiwan. ${ }^{5}$ Department of Bioresources, Da-Yeh University, Changhua, Taiwan. ${ }^{6}$ Department of Orthopedic Surgery, Center for Shockwave Medicine and Tissue Engineering, Kaohsiung Chang Gung Memorial Hospital and Chang Gung University College of Medicine; Fooyin
} 
University, School of Nursing, Kaohsiung, Taiwan. ${ }^{7}$ Department of Chinese Pharmaceutical Science and Chinese Medicine Resources, China Medical University, 91, Hsueh-Shih Road, Taichung, Taiwan.

Received: 1 August 2018 Accepted: 25 March 2019

Published online: 05 April 2019

\section{References}

1. Noteborn MH, Koch G. Chicken anaemia virus infection: molecular basis of pathogenicity. Avian Pathol. 1995;24:11-31.

2. Rosario K, Breitbart M, Harrach B, Segalés J, Delwart E, Biagini P, Varsani A. Revisiting the taxonomy of the family Circoviridae: establishment of the genus Cyclovirus and removal of the genus Gyrovirus. Arch Virol. 2017;162:1447-63.

3. Yuasa N, Taniguchi T, Yoshida I. Isolation and some characteristics of an agent inducing Anemia in chicks. Avian Dis. 1979;23:366.

4. Miller MM, Schat KA. Chicken infectious Anemia virus: an example of the ultimate host-parasite relationship. Avian Dis. 2004;48:734-45.

5. Meehan BM, Todd D, Creelan JL, Earle JA, Hoey EM, McNulty MS. Characterization of viral DNAs from cells infected with chicken anaemia agent: sequence analysis of the cloned replicative form and transfection capabilities of cloned genome fragments. Arch Virol. 1992;124:301-19.

6. Schat KA. Chicken anemia virus. Curr Top Microbiol Immunol. 2009;331:151-83.

7. Koch G, van Roozelaar DJ, Verschueren CA, van der Eb AJ, Noteborn MH. Immunogenic and protective properties of chicken anaemia virus proteins expressed by baculovirus. Vaccine. 1995;13:763-70.

8. Natesan S. Anti-neoplastic effect of chicken anemia virus VP3 protein (apoptin) in Rous sarcoma virus-induced tumours in chicken. J Gen Virol. 2006;87:2933-40.

9. Greber UF, Webster P, Weber J, Helenius A. The role of the adenovirus protease on virus entry into cells. EMBO J. 1996;15:1766-77.

10. Fletcher TM 3rd, Brichacek B, Sharova N, Newman MA, Stivahtis G, Sharp PM, Emerman M, Hahn BH, Stevenson M. Nuclear import and cell cycle arrest functions of the HIV-1 Vpr protein are encoded by two separate genes in HIV-2/SIV (SM). EMBO J. 1996;15:6155-65.

11. Lacorte C, Lohuis H, Goldbach R, Prins M. Assessing the expression of chicken anemia virus proteins in plants. Virus Res. 2007;129:80-6.

12. Cheng JH, Sheu SC, Lien YY, Lee MS, Chen HJ, Su WH. Identification of the NLS and NES motifs of VP2 from chicken anemia virus and the interaction of VP2 with mini-chromosome maintenance protein 3. BMC Vet Res. 2012;8:15.

13. Poon IK, Oro C, Dias MM, Zhang J, Jans DA. Apoptin nuclear accumulation is modulated by a CRM1-recognized nuclear export signal that is active in normal but not in tumor cells. Cancer Res. 2005;65:7059-64.

14. Lai G-H, Lien Y-Y, Lin M-K, Cheng J-H, Tzen JTC, Sun F-C, Lee M-S, Chen H-J, Lee M-S. VP2 of chicken Anaemia virus interacts with Apoptin for Downregulation of apoptosis through De-phosphorylated threonine 108 on Apoptin. Sci Rep. 2017;7.

15. Wang QM, Fan GC, Chen JZ, Chen HP, He FC. A putative NES mediates cytoplasmic localization of Apoptin in normal cells. Acta Biochim Biophys Sin. 2004;36:817-23.

16. Sun F, Pan W, Gao H, Qi X, Qin L, Wang Y, Gao Y, Wang X. Identification of the interaction and interaction domains of chicken anemia virus VP2 and VP3 proteins. Virology. 2018:513:188-94.

17. McNulty MS, Connor TJ, McNeilly F, McLoughlin MF, Kirkpatrick KS. Preliminary characterisation of isolates of chicken anaemia agent from the United Kingdom. Avian Pathol. 1990;19:67-73.

18. McNulty MS, Mackie DP, Pollock DA, McNair J, Todd D, Mawhinney KA, Connor TJ, McNeilly F. Production and preliminary characterization of monoclonal antibodies to chicken anemia agent. Avian Dis. 1990;34:352-8.

19. Scott AN, McNulty MS, Todd D. Characterisation of a chicken anaemia virus variant population that resists neutralisation with a group-specific monoclonal antibody. Arch Virol. 2001;146:713-28.

20. Trinh DQ, Yamaguchi S, Bui VN, Baatartsogt T, Imai K, Ogawa H, Kizito MK. Characterization of mAbs to chicken anemia virus and epitope mapping on its viral protein, VP1. J Gen Virol. 2015;96:1086-97.

21. Noteborn MH, Verschueren CA, Koch G, Van der Eb AJ. Simultaneous expression of recombinant baculovirus-encoded chicken anaemia virus (CAV) proteins VP1 and VP2 is required for formation of the CAV-specific neutralizing epitope. J Gen Virol. 1998;79(Pt 12):3073-7.

22. Lien YY, Huang CH, Sun FC, Sheu SC, Lu TC, Lee MS, Hsueh SC, Chen HJ. Development and characterization of a potential diagnostic monoclonal antibody against capsid protein VP1 of the chicken anemia virus. J Vet Sci. 2012;13:73-9.

23. Hu G, Zheng W, Li A, Mu Y, Shi M, Li T, Zou H, Shao H, Qin A, Ye J. A novel CAV derived cell-penetrating peptide efficiently delivers exogenous molecules through caveolae-mediated endocytosis. Vet Res. 2018;49:16.

24. Lee MS, Lien YY, Feng SH, Huang RL, Tsai MC, Chang WT, Chen HJ. Production of chicken anemia virus (CAV) VP1 and VP2 protein expressed by recombinant Escherichia coli. Process Biochem. 2009;44:390-5.

25. Thompson JD, Higgins DG, Gibson TJ. CLUSTAL W: improving the sensitivity of progressive multiple sequence alignment through sequence weighting, position-specific gap penalties and weight matrix choice. Nucleic Acids Res. 1994;22:4673-80.

26. Brown NP, Leroy C, Sander C. MView: a web-compatible database search or multiple alignment viewer. Bioinformatics. 1998;14:380-1.

27. Horton P, Park KJ, Obayashi T, Fujita N, Harada H, Adams-Collier CJ, Nakai K. WoLF PSORT: protein localization predictor. Nucleic Acids Res. 2007;35: W585-7.

28. Nguyen Ba AN, Pogoutse A, Provart N, Moses AM. NLStradamus: a simple hidden Markov model for nuclear localization signal prediction. BMC bioinformatics. 2009;10:202.

29. la Cour T, Kiemer L, Molgaard A, Gupta R, Skriver K, Brunak S. Analysis and prediction of leucine-rich nuclear export signals. Protein Eng, Des Sel. 2004; 17:527-36.

30. Gould CM, Diella F, Via A, Puntervoll P, Gemund C, Chabanis-Davidson S, Michael S, Sayadi A, Bryne JC, Chica C, et al. ELM: the status of the 2010 eukaryotic linear motif resource. Nucleic Acids Res. 2010;38:D167-80.

31. Todd D. Circoviruses: immunosuppressive threats to avian species: a review. Avian Pathol. 2000;29:373-94.

32. Todd D, Creelan JL, Mackie DP, Rixon F, McNulty MS. Purification and biochemical characterization of chicken anaemia agent. J Gen Virol. 1990; 71(Pt 4):819-23.

33. Meehan BM, MCNeilly F, Todd D, Kennedy S, Jewhurst VA, Ellis JA, Hassard LE Clark EG, Haines DM, Allan GM. Characterization of novel circovirus DNAs associated with wasting syndromes in pigs. J Gen Virol. 1998;79(Pt 9):2171-9.

34. Phan TG, Li L, O'Ryan MG, Cortes H, Mamani N, Bonkoungou IJ, Wang C Leutenegger CM, Delwart E. A third gyrovirus species in human faeces. J Gen Virol. 2012:93:1356-61.

35. Peters MA, Jackson DC, Crabb BS, Browning GF. Chicken anemia virus VP2 is a novel dual specificity protein phosphatase. J Biol Chem. 2002;277:39566-73.

36. Peters MA, Crabb BS, Washington EA, Browning GF. Site-directed mutagenesis of the VP2 gene of chicken anemia virus affects virus replication, cytopathology and host-cell MHC class I expression. J Gen Virol. 2006;87:823-31.

37. Yamaguchi S, Imada T, Kaji N, Mase M, Tsukamoto K, Tanimura N, Yuasa N. Identification of a genetic determinant of pathogenicity in chicken anaemia virus. J Gen Virol. 2001:82:1233-8.

38. Fischer U, Huber J, Boelens WC, Mattaj IW, Luhrmann R. The HIV-1 rev activation domain is a nuclear export signal that accesses an export pathway used by specific cellular RNAs. Cell. 1995;82:475-83.

39. Wen W, Meinkoth JL, Tsien RY, Taylor SS. Identification of a signal for rapid export of proteins from the nucleus. Cell. 1995;82:463-73.

40. Cao S, Liu X, Yu M, Li J, Jia X, Bi Y, Sun L, Gao GF, Liu W. A nuclear export signal in the matrix protein of influenza a virus is required for efficient virus replication. J Virol. 2012;86:4883-91.

41. Huang S, Chen J, Chen Q, Wang H, Yao Y, Chen J, Chen Z. A second CRM1-dependent nuclear export signal in the influenza a virus NS2 protein contributes to the nuclear export of viral ribonucleoproteins. $J$ Virol. 2013;87:767-78.

42. Onder Z, Chang V, Moroianu J. Nuclear export of cutaneous HPV8 E7 oncoprotein is mediated by a leucine-rich nuclear export signal via a CRM1 pathway. Virology. 2015:474:28-33.

43. Booth DS, Cheng Y, Frankel AD. The export receptor $\mathrm{Crm} 1$ forms a dimer to promote nuclear export of HIV RNA. Elife. 2014;3:e04121.

44. Heilman DW, Teodoro JG, Green MR. Apoptin nucleocytoplasmic shuttling is required for cell type-specific localization, apoptosis, and recruitment of the anaphase-promoting complex/cyclosome to PML bodies. J Virol. 2006;80:7535-45. 